\title{
On the influence of particle concentration in a lubricant and its rheological properties on the bearing behavior
}

\author{
Benyebka Bou-Said ${ }^{1, a}$, Hamid Boucherit ${ }^{2}$ And Mustapha Lahmar ${ }^{2}$ \\ 1 Université de Lyon, CNRS INSA-Lyon, LaMCoS, UMR 5259, 69621 Villeurbanne, France \\ 2 Mechanical Engineering Department, Faculty of Sciences and Technology Guelma University, Algeria
}

Received 9 October 2011, Accepted 26 January 2012

\begin{abstract}
Vibration and wear debris analyses are the two main conditions monitoring techniques for machinery maintenance and fault diagnosis. In addition, numerical simulation can provide useful tools to assess the proper functioning of industrial machines. We use different suspension theories with a concern of the effect of solid particles liquid lubricant itself on bearing behavior. We consider both simple models based on the Einstein's mixture theory and a micropolar fluid theory, which is characterized by the presence of suspended rigid micro-structured particles. We suppose in this study that there are no abrasion and no wear (which are not beneficial for the contact performances) due to the presence of particles. Thus in this case we found theoretically that the presence of rigid particles in the lubricant increases the effective viscosity which enhances the load-carrying capacity as well as the minimum film thickness.
\end{abstract}

Key words: Non-Newtonian fluid / particles / pollution

\section{Introduction}

In recent years there appears to have been a growing interest in the study of the importance of non-Newtonian effects in lubrication problems. The problem of nonNewtonian lubrication theory mainly reduces to the determination of a realistic equation of state (constitutive equation) connecting stress and rate of deformation. It is also essential that the equation chosen shall be simple and mathematically permissible in the sense of invariance. Most investigations to date, while using mathematically simple models, have used either physically unrealistic or mathematically inadmissible equations of state; and it appears that a re-appraisal is necessary. The presence of nonlinearities during the simple shear flow of such lubricant is considered to increase the load carrying capacity of the lubricant film whereas the reduction in the viscosity of the lubricant with shear rate contributes to a reduction in friction. The non-Newtonian behavior of the lubricant is found to delay the onset of turbulence and cavitation and to stabilize the flow. The formulation of a single generalized constitutive equation for the investigation of different types of flow situations is almost impossible. An adequate form of the constitutive equation which could satisfactorily predict the required aspects of the flow situation to

\footnotetext{
a Corresponding author:

benyebka.bou-said@insa-lyon.fr
}

the required degree of accuracy is the actual requirement for investigation [1-3].

A simple approach consists in considering the lubricant as a fluid containing particles of given size and concentration. This elementary phenomenological approach can be used when studying, for example, the effects of contamination. Another approach used to characterize these fluids consists in considering these lubricants as polar fluids. Physically, the polar fluids consist of rigid, randomly oriented particles suspended in a viscous medium. Mineral oils containing a small amount of particles are the examples of couple-stress fluids. The main particularity of couple-stress fluids is that the stress tensor is antisymmetric.

In order to better describe the flow behavior of this kind of non-Newtonian fluid, different micro-continuum theories were therefore developed (Stokes [4], Ariman and Sylvester [5], Ariman and Sylvester [6]). Among these theories, the couple-stress fluid model proposed first by Stokes [4] is the simplest and has been widely used. The incompressible couple-stress fluid is physically characterized by two constants, namely viscosity $\mu$ and couple stress parameter $\eta$. Note that the new material constant $\eta$ is responsible for the couple-stress property. In the literature, the effects of couple-stresses on the behavior of journal bearings are theoretically studied by defining the dimensionless couple-stress parameter (whose the value ranges from zero to one), having the dimension of length. 


\section{Nomenclature}

\begin{tabular}{|c|c|}
\hline $\bar{C}$ & bearing radial clearance, $(\mathrm{m})$ \\
\hline$h$ & fluid-film thickness, (m) \\
\hline$F_{X}, F_{Y}$ & components of the hydrodynamic carrying capacity \\
\hline$\tilde{F}_{X}, \tilde{F}_{Y}$ & dimensionless components of the hydrodynamic carrying capacity, $\left(\tilde{F}_{X}, \tilde{F}_{Y}\right)=\frac{\left(F_{X}, F_{Y}\right)}{\mu \omega R L\left(\frac{R}{C}\right)^{2}}$ \\
\hline$f$ & friction factor where $F_{c}$ is the friction force on the bush and $W_{0}$ the static load $f=\frac{F_{c}}{\tilde{W}_{0}}$ \\
\hline$L$ & bearing length, $(\mathrm{m})$ \\
\hline$\ell$ & characteristic length of additives, $(\ell=\sqrt{(\eta / \mu)}),(\mathrm{m})$ \\
\hline$\tilde{\ell}$ & couple-stress parameter, $(\tilde{\ell}=\ell / C)$ \\
\hline$N_{\theta}$ & number of cells in circumferential direction \\
\hline$N_{\mathrm{z}}$ & number of cells in the axial direction \\
\hline$\tilde{Q}$ & dimensionless total mean leakage flow, $\left(\tilde{Q}=Q L /\left(\omega C R^{3}\right)\right)$ \\
\hline$K_{i j}$ & rate of rotation tensor \\
\hline$p$ & fluid-film pressure, $(\mathrm{Pa})$ \\
\hline$\tilde{p}$ & normalized film pressure, $\left(\tilde{p}=\frac{p}{\mu \omega(R / C)^{2}}\right)$ \\
\hline$R$ & journal radius, $(\mathrm{m})$ \\
\hline$t$ & time, $(\mathrm{s})$ \\
\hline$u, v, w$ & velocity components in circumferential, radial and axial directions, respectively, $\left(\mathrm{m} . \mathrm{s}^{-1}\right)$ \\
\hline$\tilde{W}$ & dimensionless load carrying-capacity, $\tilde{W}=\left(\tilde{F}_{x}^{2}+\tilde{F}_{y}^{2}\right)^{1 / 2}$ \\
\hline$z$ & Cartesian coordinate axis along the bearing axis, $(\mathrm{m})$ \\
\hline$\tilde{z}$ & dimensionless axial coordinate \\
\hline$\left(0_{c}, \varepsilon, \phi\right)$ & $\begin{array}{l}\text { Cartesian coordinate system centered at } 0_{\mathrm{c}} \text { with unit vectors } \varepsilon \text { and } \phi \text { parallel and } \\
\text { perpendicular, respectively, to the center line }\end{array}$ \\
\hline$\left(0_{c}, X, Y\right)$ & coordinate system related to the static load \\
\hline$\varepsilon$ & static eccentricity ratio, $(\varepsilon=e / C)$ \\
\hline$e$ & static eccentricity, $\left(e=\left|0_{\mathrm{c}} 0_{a}\right|\right),(\mathrm{m})$ \\
\hline$\eta$ & material constant responsible for the couple-stress property, (N.s) \\
\hline$\phi$ & bearing attitude angle, (rad) \\
\hline$\mu$ & absolute viscosity of the lubricant, (Pa.s) \\
\hline$\mu_{s}$ & viscosity of the solvent \\
\hline$\theta$ & bearing angle with the origin at the maximum film thickness, (rad) \\
\hline$\theta_{c 0}$ & static cavitation angle, (rad) \\
\hline$\omega$ & journal angular velocity, (rpm) \\
\hline$\Phi$ & volumic concentration, \\
\hline$e_{b}$ & unbalance eccentricity, $(\mathrm{m})$ \\
\hline$M$ & rotor mass supported by the bearing, $(\mathrm{kg})$ \\
\hline$O_{a}$ & shaft center, \\
\hline$O_{\mathrm{c}}$ & bearing center, \\
\hline$X, Y$ & shaft center coordinates, $(\mathrm{m})$ \\
\hline$\tilde{X}, \tilde{Y}$ & dimensionless shaft center coordinates, $(\tilde{X}, \tilde{Y})=(X, Y) / C$ \\
\hline$\Phi_{\mathrm{m}}$ & acceptable maximum concentration \\
\hline$\varepsilon_{b}$ & unbalance eccentricity ratio, $\varepsilon_{b}=e_{b} / C$ \\
\hline
\end{tabular}

It can be thought of as a fluid property depending on the size of the particle. Owing to its relative mathematical simplicity, the Stokes' couple-stress fluid model has been widely applied to analyze various hydrodynamic and hydrostatic lubrication problems. Mokhiamer et al. [7] presented an excellent theoretical study of the performance of a steadily-loaded compliant journal bearing using couplestress fluid as lubricant. The results obtained are:

- both the maximum pressure and load-carrying capacity increase with the increase of the couple-stress parameter;

- the attitude angle and the friction factor decrease when the couple-stress parameter increases;
- the side leakage flow remains almost the same as the couple-stress parameter increases.

In this paper we investigate the effects of particles in a lubricant on bearing behavior. A special attention is paid to the influence of particle concentration.

\section{Rheological law}

The simplest law of rheological behavior is that of Newtonian type. The relationship between the shearing and the velocity gradient across the film is linear when considering the thin film theory (Fig. 1). Thus only the 


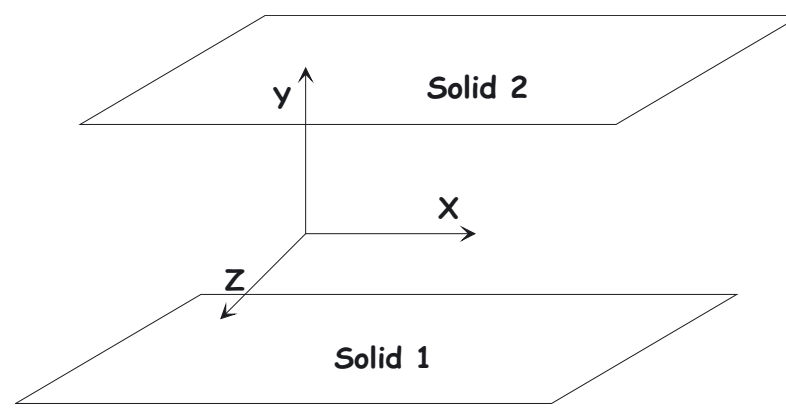

Fig. 1. Contact scheme.

shear stresses $\tau_{x y}$ and $\tau_{z y}$ are taken into account and the constitutive laws are written:

$$
\left\{\begin{array}{l}
\tau_{x y}=\mu \frac{\partial u}{\partial y} \\
\tau_{z y}=\mu \frac{\partial w}{\partial y}
\end{array}\right.
$$

where:

$\mu$ : dynamic viscosity of the fluid,

$u$ and $w$ : velocity components of a fluid particle according to $x$ and $z$ axes respectively.

$\tau_{x y}$ and $\tau_{z y}$ : viscous shear stresses.

For a Newtonian and conpressible fluid, the relation linking the shearing $\left(\sigma_{i j}\right)$ to the tensor of rate of deformation $\left(D_{i j}\right)$ is written:

$$
\sigma_{i j}=(-p+\lambda \Theta) \delta_{i j}+2 \mu D_{i j}
$$

with,

$p$ : static pressure,

$D_{i j}=\frac{1}{2}\left(u_{i, j}+u_{j, i}\right)$ : the tensor of rate of deformation,

$\Theta$ : cubic dilatation $\left(\Theta=D_{k k}=\frac{\partial u_{k}}{\partial x_{k}}\right)$,

$\delta_{i j}$ : Kronecker delta (unit tensor),

$\lambda$ and $\mu$ : viscous fluid parameters (Navier coefficients).

For an incompressible fluid $(\Theta=0)$, the second coefficient of viscosity $\lambda$ does not play a role.

The presence of particles leads to non-Newtonian rheological behavior. Several approaches are possible and discussed below.

\subsection{Suspension of solid spherical particles}

The simplest method to represent the macroscopic structure of a fluid with particles is to consider the mixture as a suspension of rigid spherical particles in a Newtonian solvent. The rheological behavior is still Newtonian [7]. The only change concerns the dynamic viscosity. The predicted change in viscosity is based on so-called dilute solutions theory [8] which began in 1906 due to Einstein. The theory has been broadly modified by other authors. The simplest and the most widely used variations are as follows:

\section{(a) Model of Einstein}

Einstein's work in rheology concerns very dilute suspensions of rigid spheres with volumic concentration $\Phi$, in a Newtonian solvent of dynamic viscosity $\mu_{s}$. He proposed the following expression, valid for any type of fluid flow:

$$
\frac{\mu}{\mu_{s}}=1+2.5 \times \Phi
$$

where:

$\frac{\mu}{\mu_{s}}$ : average relative viscosity of the suspension $\mu$ : fluid viscosity $\mu_{s}$ : viscosity of the solvent.

\section{(b) Model of Batchelor and Green}

In 1972, Batchelor and Green, added a quadratic term to the Einstein's expression, to take into account the Brownian movement of the particles $[8,9]$. This refers to the random action of the lubricant on the particles. The previous expression (3) becomes:

$$
\frac{\mu}{\mu_{s}}=1+2.5 \times \Phi+6.2 \times \Phi^{2}
$$

\section{(c) Model of Ball and Richmond}

In 1980, Ball and Richmond changed the model of Batchelor and Green, by introducing an acceptable maximum concentration $\left(\Phi_{\mathrm{m}} \approx 0.64\right.$ for spheres in solvent) $[8,9]$ :

$$
\frac{\mu}{\mu_{s}}=\left(1-\frac{\Phi}{\Phi_{m}}\right)^{-2.5 \times \Phi_{m}}
$$

\section{(d) Model of Phan-Thien and Pham}

Phan-Thien and Pham, in 1987 offer a different expression of the fluid viscosity which is given by the following expression $[8,9]$ :

$$
\frac{\mu}{\mu_{s}}=(1-\Phi)^{-2.5}
$$

\subsection{Couple stress fluid (polar fluid)}

Most oils used for the lubrication of rotating machinery contain particles and/or additives. These particles and additives cause modification to the rheological behavior of the resulting fluid. According to the continuum theory of Vijay Kumar Stokes (Micro Continuum Theory) [4], the behavioral law for this fluid (called a complex fluid or polar fluid) can be written as:

$$
\sigma_{i j}=-p \delta_{i j}+\mu\left(u_{i, j}+u_{j, i}\right)-\frac{1}{2} e_{i j k} M_{r k, r}
$$

with: $M_{i j}=\frac{1}{3} M_{n n} \delta_{i j}+4 \eta K_{i j}$,

$\sigma_{i j}$ : non symmetrical stress tensor,

$M_{r k}$ : couple stress tensor,

$e_{i j k}$ : permutation tensor of order three,

$K_{i j}$ : rate of rotation tensor,

$\mu$ : coefficient of viscosity with a dimension of $[\mu]=$ $M L^{-1} T^{-1}$,

$\eta$ : physical constant due to the presence of the couple stress with a dimension of $[\eta]=M L T^{-1}$. 


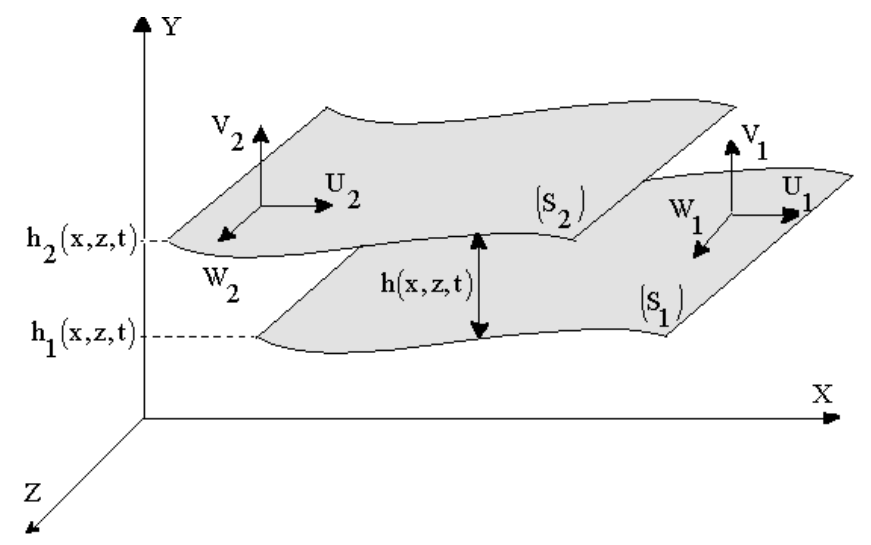

Fig. 2. Geometry and kinematics of contact.

\section{Reynolds equation}

For a couple stress isoviscous fluid (Fig. 2), the modified Reynolds equation is given in the following form [7]:

$$
\begin{gathered}
\frac{\partial}{\partial x}\left[G(h, \ell) \frac{\partial p}{\partial x}\right]+\frac{\partial}{\partial z}\left[G(h, \ell) \frac{\partial p}{\partial z}\right]= \\
6 \mu\left(\left(U_{2}+U_{1}\right) \frac{\partial h}{\partial x}-2 U_{2} \frac{\partial h}{\partial x}+\left(W_{2}+W_{1}\right) \frac{\partial h}{\partial z}-2 U_{2} \frac{\partial h}{\partial x}\right. \\
\left.-2 W_{2} \frac{\partial h}{\partial z}+2 V_{2}\right)
\end{gathered}
$$

with: $G(h, \ell)=h^{3}-12 \ell^{2} h+24 \ell^{3} \tanh \left(\frac{h}{2 \ell}\right)$.

In this equation:

$p(x, z, t)$ : pressure in the film

$h(x, z, t):$ thickness of the film

$t$ : time

$\mu$ : constant fluid viscosity

$\ell$ : couple stress parameter which physically represents the size of the particle and can be expressed as $\ell=\sqrt{\eta / \mu}$ where $\eta$ is a physical constant of the lubricant due to the presence of couple stresses [4].

$U_{1}$ and $U_{2}$ speeds of solids, $S_{1}$ and $S_{2}$ in the the $x$ direction, $W_{1}$ and $W_{2}$ speeds of solids, $S_{1}$ and $S_{2}$ in the $z$-direction.

In hydrodynamic regime, the modified Reynolds Equation (8) for a lubricated bearing with a couple stress fluid becomes [7]:

$$
\begin{aligned}
\frac{1}{R^{2}} \frac{\partial}{\partial \theta}\left[G(h, \ell) \frac{\partial p}{\partial \theta}\right]+\frac{\partial}{\partial z}[G & \left.(h, \ell) \frac{\partial p}{\partial z}\right] \\
& =6 \mu \omega \frac{\partial h}{\partial \theta}+12 \mu \frac{\partial h}{\partial t}
\end{aligned}
$$

In dimensionless form this equation becomes:

$$
\begin{aligned}
\frac{\partial}{\partial \theta}\left[\tilde{G}(\tilde{h}, \tilde{\ell}) \frac{\partial \tilde{p}}{\partial \theta}\right]+\left(\frac{R}{L}\right)^{2} \frac{\partial}{\partial \tilde{z}}[ & \left.\tilde{G}(\tilde{h}, \tilde{\ell}) \frac{\partial \tilde{p}}{\partial \tilde{z}}\right] \\
& =6 \frac{\partial \tilde{h}}{\partial \theta}+12 \frac{\partial \tilde{h}}{\partial \tilde{t}}
\end{aligned}
$$

with: $\tilde{G}(\tilde{h}, \tilde{\ell})=\tilde{h}^{3}-12 \tilde{\ell}^{2} \tilde{h}+24 \tilde{\ell}^{3} \tanh \left(\frac{\tilde{h}}{2 \tilde{\ell}}\right)$ for a newtonian fluid $\left(\tilde{\ell}=0: \tilde{G}=\tilde{h}^{3}\right)$ and: $\theta=\frac{x}{R}, \tilde{z}=\frac{z}{L}, \tilde{h}=\frac{h}{C}$, $\tilde{t}=\omega t, \tilde{\ell}=\frac{\ell}{C}$ and $\tilde{p}=\frac{p}{\mu \omega\left(\frac{R}{C}\right)^{2}}$.

The film thickness (Fig. 3a) is given as: $h=C+$ $x(t) \cos \theta+y(t) \sin \theta$,

$\omega$ : rotational speed of the rotor,

$C$ : radial clearance of the bearing.

$R$ and $L$ are the radius and the length of the bearing, respectively.

The Reynolds equation for a Newtonian fluid as well as the modified Reynolds equation must be solved numerically. We use here the finite difference method. The obtained nonlinear algebraic system of equations is solved using iterative method of Gauss-Seidel with an over-relaxation coefficient according to the algorithm of Christopherson which allows us to take into consideration the film rupture.

Obtaining the pressure field we can calculate the components of the hydrodynamic carrying capacity:

$$
\left\{\begin{array}{l}
F_{x} \\
F_{y}
\end{array}\right\}=\int_{-L / 2}^{L / 2} \int_{\theta_{1}}^{\theta_{2}} p(\theta, z, t)\left\{\begin{array}{c}
\cos \theta \\
\sin \theta
\end{array}\right\} R \mathrm{~d} \theta \mathrm{d} z
$$

where $\theta_{1}$ and $\theta_{2}$ are the angular locations of rupture and film reformation, respectively.

The following boundary conditions have been applied:

$$
\begin{aligned}
& \tilde{p}_{0}=0 \quad \text { at } \quad \tilde{z}= \pm \frac{1}{2} \\
& \tilde{p}=\frac{\partial \tilde{p}}{\partial \theta}=0 \quad \text { at } \quad \theta=\theta_{c 0}
\end{aligned}
$$

Equation (11) results from the fact that the ends of the bearing are exposed to the atmospheric pressure, while equation (12) is the Swift-Stieber conditions known as the Reynolds conditions. $\theta_{c 0}$ is the film rupture angle, so-called the cavitation angle measured from the maximum film thickness at which cavitation starts. It is not a prescribed value but is determined during calculations by applying the Christopherson's algorithm [10].

The dimensionless total mean leakage flow of the journal bearing is expressed by:

$$
\tilde{Q}=\left|\tilde{Q}_{+}\right|+\left|\tilde{Q}_{-}\right|
$$

where

$$
\tilde{Q}_{+}=\int_{0}^{2^{\pi}}[\tilde{h} \tilde{\bar{w}}]_{\tilde{z}=1 / 2} \mathrm{~d} \theta \text { and } \tilde{Q}_{-}=\int_{0}^{2^{\pi}}[\tilde{h} \tilde{\bar{w}}]_{\tilde{z}=-1 / 2} \mathrm{~d} \theta
$$

$\tilde{Q}_{+}$and $\tilde{Q}_{-}$are the dimensionless mean leakage flows at each end of the journal bearing.

The dimensionless mean velocities of the flow are given by:

$$
\left\{\begin{array}{l}
\tilde{\bar{u}}=\frac{1}{2}-\frac{\tilde{h}^{2}}{12} \frac{\partial \tilde{p}}{\partial \theta}+\tilde{\ell}^{2} \frac{\partial \tilde{p}}{\partial \theta}\left(1-\frac{2 \tilde{\ell}}{\tilde{h}} \tanh \left(\frac{\tilde{h}}{2 \tilde{\ell}}\right)\right) \\
\tilde{\bar{w}}=-\frac{\tilde{h}^{2}}{12} \frac{\partial \tilde{p}}{\partial z}+\tilde{\ell}^{2} \frac{\partial \tilde{p}}{\partial z}\left(1-\frac{2 \tilde{\ell}}{\tilde{h}} \tanh \left(\frac{\tilde{h}}{2 \tilde{\ell}}\right)\right)
\end{array}\right.
$$




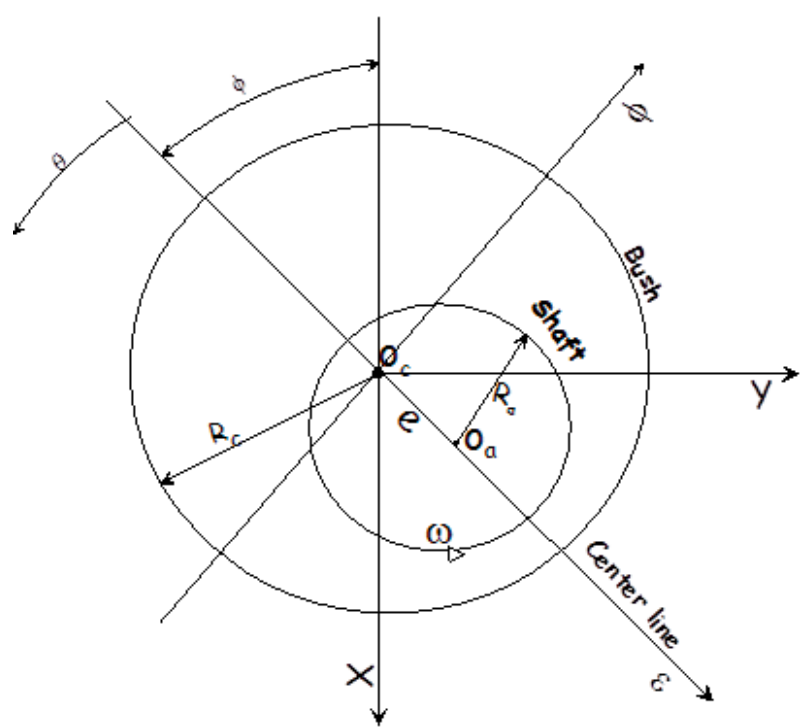

(a)

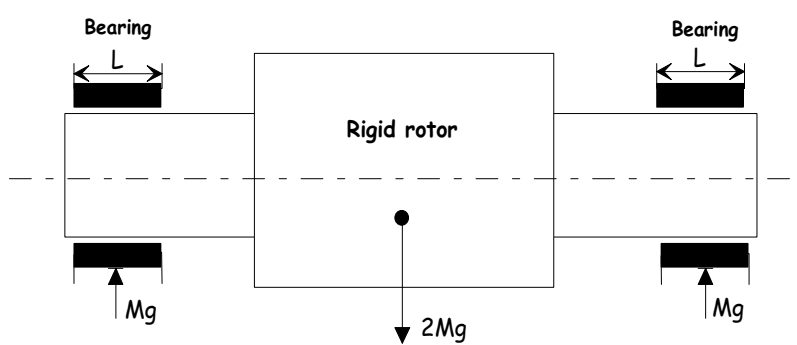

(b)

Fig. 3. Rotor-bearing system and system axes. (a) Crosssection of the bearing. (b) Schematic of the system.

\section{Method of resolution}

\subsection{Method of solution in steady-state conditions}

The static solution of hydrodynamic problem is obtained by an iterative numerical procedure. The following steps are performed:

1. Select the input parameters of the problem $\varepsilon, R, L, \tilde{\ell}$, $\Phi, \omega, \mu, N_{0}, N_{z}$, relaxation factors, convergence criteria and maximum number of iterations for the pressure. $\left(N_{\theta}\right.$ cells in circumferential direction, $N_{z}$ cells in the axial direction.)

2. Compute the film thickness $\tilde{h}$.

3. Initialize the iteration number $n$ to 0 .

4. Initialize the dimensionless static pressure field $\tilde{p}^{(n)}=$ 0 .

5. Solve the modified Reynolds equation for the pressure field $\tilde{p}^{(n+1)}$ using the finite difference method with successive over-relaxation scheme. The iterative process is repeated until either the pressure convergence criterion is achieved or the maximum number of iterations is exceeded.

6. Check the static film pressure convergence criterion $\frac{1}{N} \sum_{i, j}\left|\frac{\tilde{p}_{i j}^{(n+1)}-\tilde{p}_{i j}^{(n)}}{\tilde{p}^{(n+1)}}\right| \leq 10^{-5}$ where $N$ is the total number of nodes for which the pressure is positive. If convergence is not achieved, then increment the iteration number $n$ by 1 and return to step 5 .

7. Calculate the static performance characteristics.

\subsection{Equations of rotor motion and method of resolution}

The different forces which act on the shaft are the weight $\mathrm{Mg}$, the hydrodynamic forces $F_{X}$ and $F_{Y}$, the inertia forces $M \ddot{X}$ and $M \ddot{Y}$ and the unbalance forces characterized by an eccentricity $e_{b}$ (Fig. $3 \mathrm{~b}$ ). The application of Newton's second law to the shaft movement allows us to write:

$$
\left\{\begin{array}{l}
M \ddot{X}=M g+F_{x}(X, Y, \dot{X}, \dot{Y})+M e_{b} \omega^{2} \cos (\omega t) \\
M \ddot{Y}=F_{y}(X, Y, \dot{X}, \dot{Y})+M e_{b} \omega^{2} \sin (\omega t)
\end{array}\right.
$$

In Equation (15) the hydrodynamic forces are nonlinear functions of coordinates $X$ and $Y$ and speeds $\dot{X}$ and $\dot{Y}$ of the shaft center. Initial conditions are chosen arbitrarily. After integrating the nonlinear differential Equations (15) by an explicit Euler method, we can calculate displacements $X(t)$ and $Y(t)$ of the shaft center according to the following scheme:

$$
\left\{\begin{array}{l}
\dot{X}(t+\Delta t)=\dot{X}(t)+\ddot{X}(t) \Delta t \\
X(t+\Delta t)=X(t)+\dot{X}(t+\Delta t) \Delta t
\end{array}\right.
$$

and similar equations for the $Y$ displacement and the speed $\dot{X}$ and $\dot{Y}$.

\section{Parametric study}

The parametric study in this work concerns a comparative investigation of a bearing lubricated by: a Newtonian fluid, a polar fluid (couple stress fluid), and a fluid with spherical solid particles (the effect of particle concentration). The results concern:

(1) The shaft center trajectories of a bearing subject to a static load (e.g., weight) and/or its dynamic response (e.g., unbalance).

(2) The variations of minimum film thickness and maximum pressure in the film as functions of time, which are both important parameters to consider in design of the bearing.

(3) The effect of the fluid rheology on the nonlinear dynamic behavior of the bearings.

The following data have been used (Tab. 1).

\subsection{Static performance}

Figures 4 to 8 depict, respectively, the dimensionless pressure distribution for $\varepsilon=0.7$, dimensionless carrying capacity, dimensionless side leakage flow, friction factor, 
Table 1. Bearing geometry, running conditions and lubricant properties.

\begin{tabular}{lc}
\hline Geometry & $35 \times 10^{-3} \mathrm{~m}$ \\
- bearing length, $L$ & $35 \times 10^{-3} \mathrm{~m}$ \\
- shaft radius, $R$ & $25 \times 10^{-5} \mathrm{~m}$ \\
- radial clearance, $C$ & \\
Running conditions & $54 \mathrm{~kg}$ \\
- rotor mass allocated to the bearing, $M$ & $5000 \mathrm{rpm}$ \\
- rotating speed, $N$ & \\
Lubrifiant properties, & $0.001 \mathrm{Pa.s}$ \\
- dynamic viscosity at atmospheric pressure, $\mu$ & $1000 \mathrm{~kg} . \mathrm{m}^{-3}$ \\
- density, $\rho$ & $0.0,0.1$ and 0.3 \\
\hline
\end{tabular}
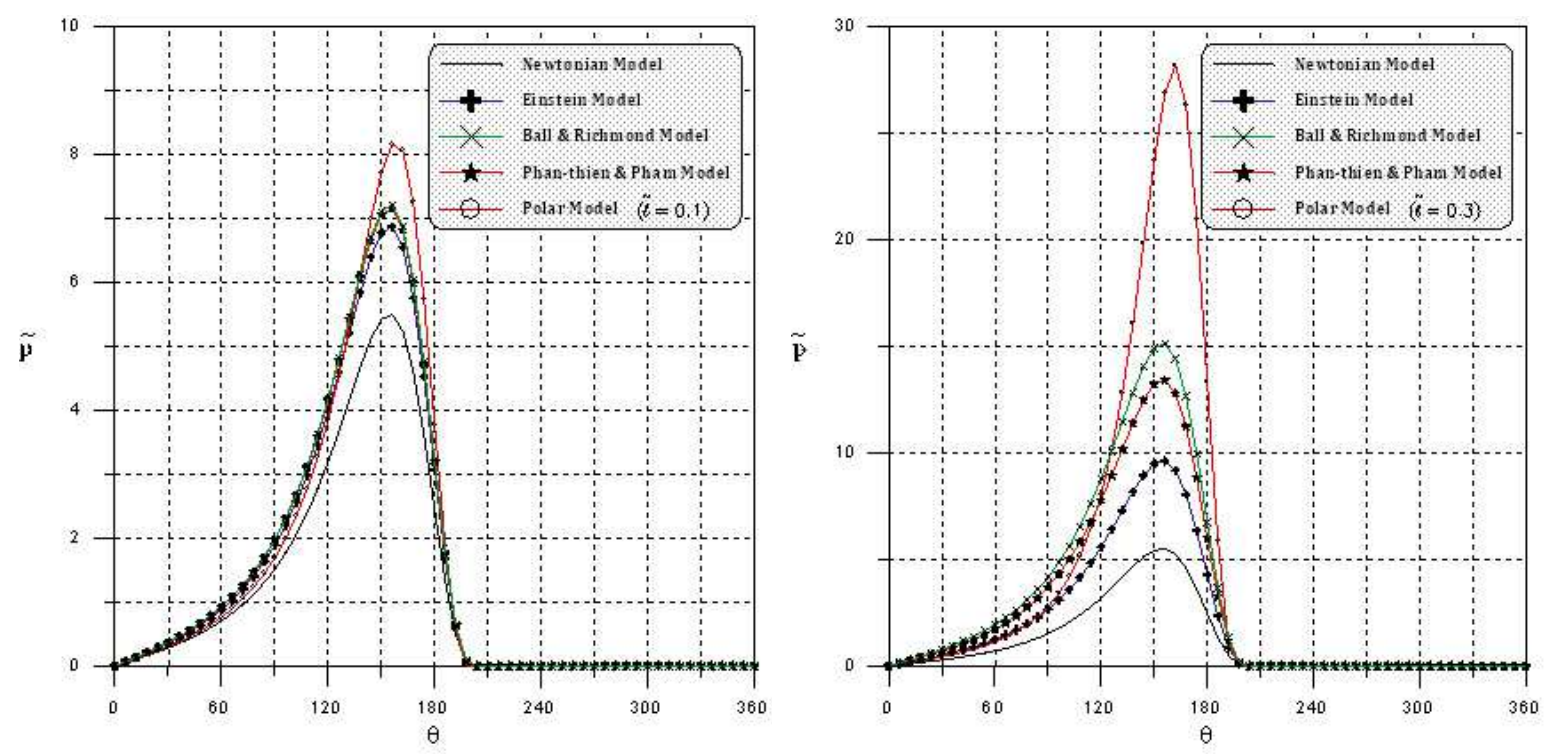

Fig. 4. Dimensionless pressure distribution for different rheological models $(\Phi=10 \%$ left, and $\Phi=30 \%$ right; $\varepsilon=0.7)$.

and attitude angle versus eccentricity ratio; for two values of the particle concentration, namely, $10 \%$ and $30 \%$. Several comments can be made. Increasing the concentration of particles increases the apparent viscosity, thus leading to a higher pressure. The values obtained depend on the model and its complexity. Incidentally, the non-Newtonian models give similar dimensionless load at small particle concentration which is higher when compared to the Newtonian case. For higher values of particle concentration there is a differentiation between the different models, the Einstein model predicting a more less extent of non-linearity. A significant increase of the dimensionless side leakage flow is observed when using Einstein law or related models. We do not observe any noticeable change with the polar model when compared to the Newtonian case.

The same tendencies are observed for the friction factor in which the attitude angle does not present any notable change. These latter results are in good agreements with those from Mokhiamer et al. [7] and Boucherit et al. [11] who show that: both maximum pressure and load-carrying capacity increase with the increase of the couple-stress parameter, the attitude angle and friction factor decrease when the couple-stress parameter increases, and the side leakage flow remains almost constant as the couple-stress parameter increases.

\subsection{Dynamic response}

Figures 9 and 10 show the evolution of the minimum film thickness and maximum pressure with time for $10 \%$ concentration of particles in a solvent. We note that the presence of particles increases markedly the minimum film thickness and slightly decreases the maximum pressure as the effective viscosity rises. The couple stress model gives higher values of pressure as it takes into account more phenomena in describing flow resistance.

The equilibrium position of the shaft is stable as shown in Figure 11 which represents the shaft trajectories for different rheological models.

We obtain the same tendencies when increasing the concentration of particles (20\% and $30 \%$ in Figs. 12 to 17 ) with an enlargement of the response. We note that when the concentration of particles increases, the bearing behaviour can become unstable. This is consistent with the results presented in Figure 8 where it has been noted that the attitude angle increases with particles concentration. 

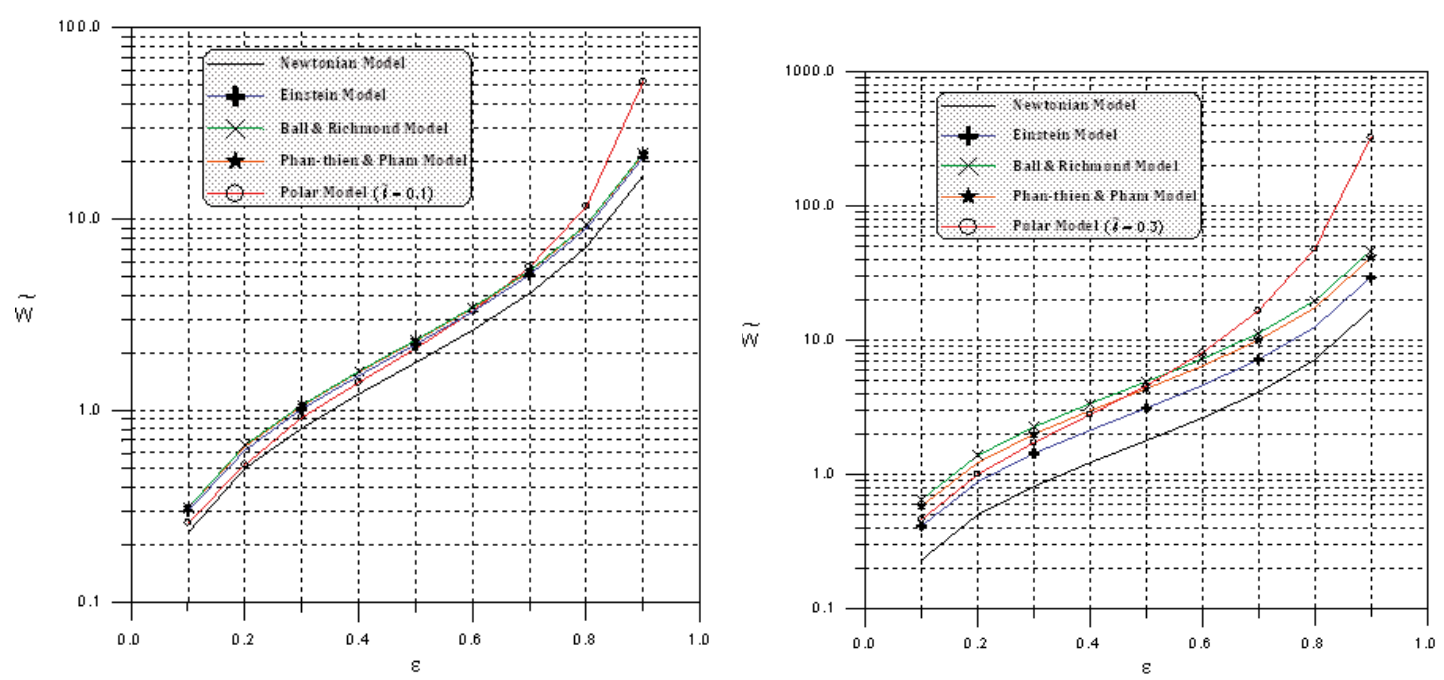

Fig. 5. Dimensionless carrying capacity versus eccentricity ratio for different rheological models $(\Phi=10 \%$ left, and $\Phi=30 \%$ right).
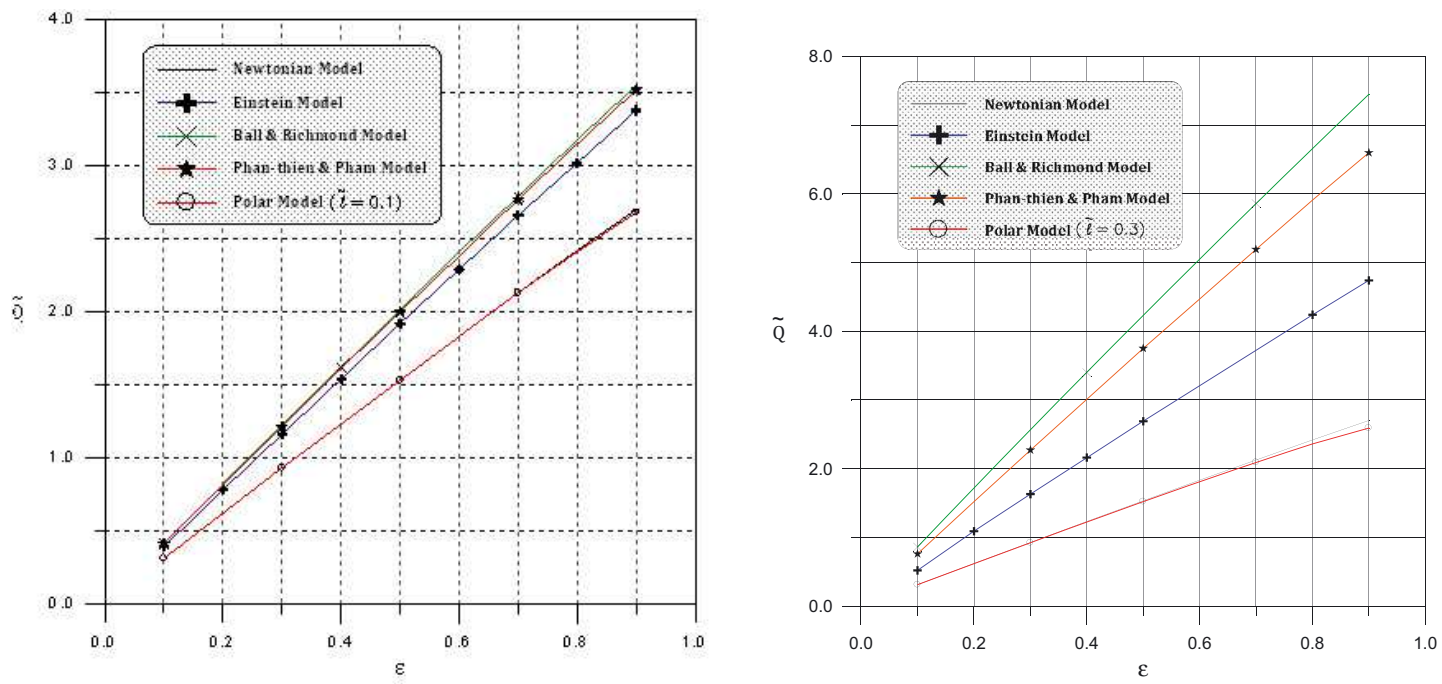

Fig. 6. Dimensionless side leakage flow versus static eccentricity ratio for different rheological models $(\Phi=10 \%$ left, and $\Phi=30 \%$ right).
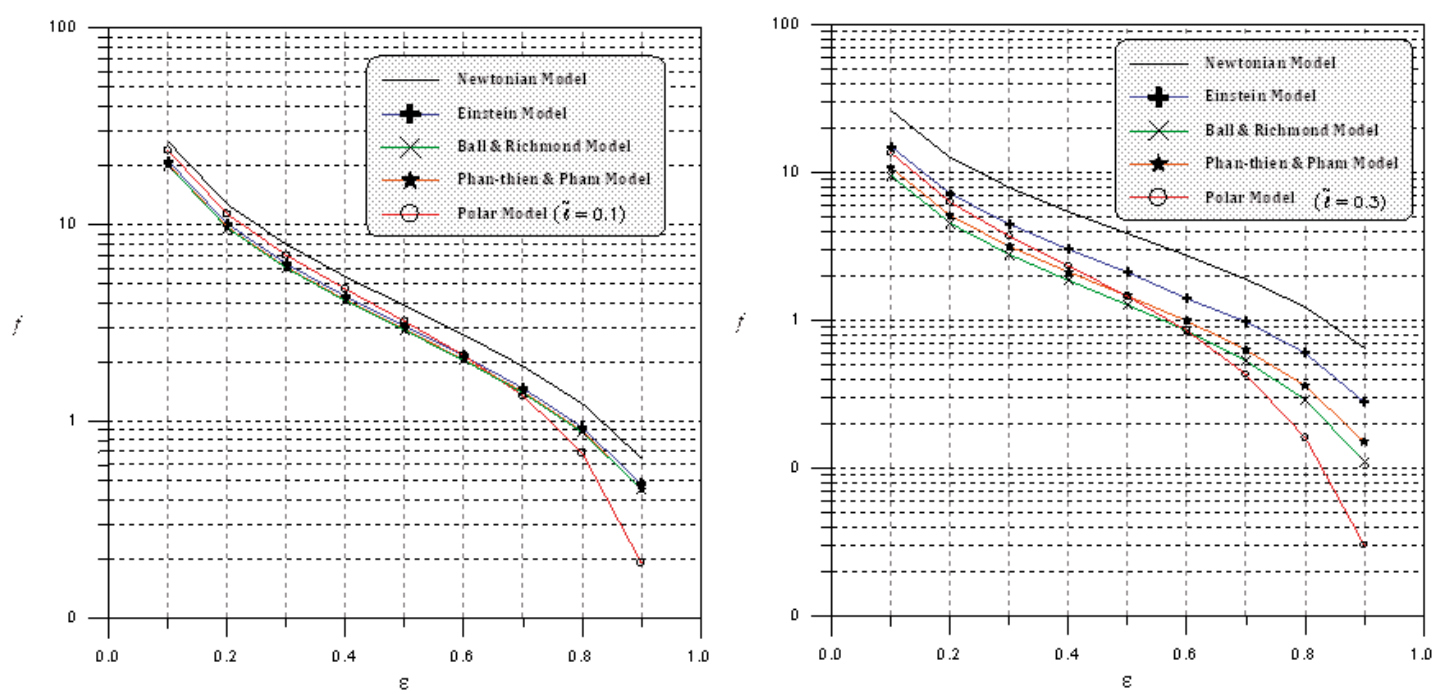

Fig. 7. Friction factor versus static eccentricity ratio for different rheological models $(\Phi=10 \%$ left and $\Phi=30 \%$ right $)$. 

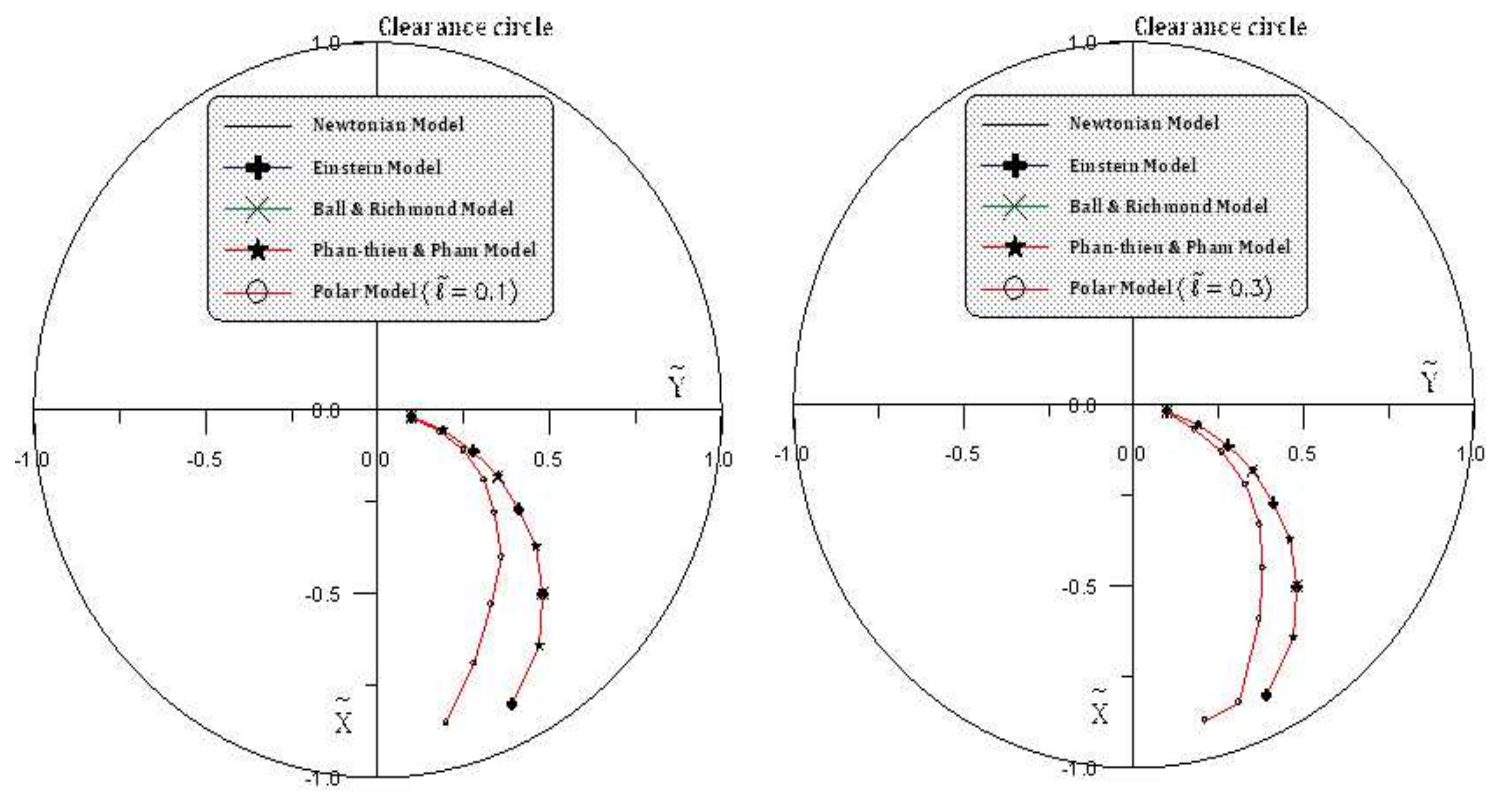

Fig. 8. Static attitude angle versus static eccentricity ratio for different rheological models $(\Phi=10 \%$ left and $\Phi=30 \%$ right $)$.

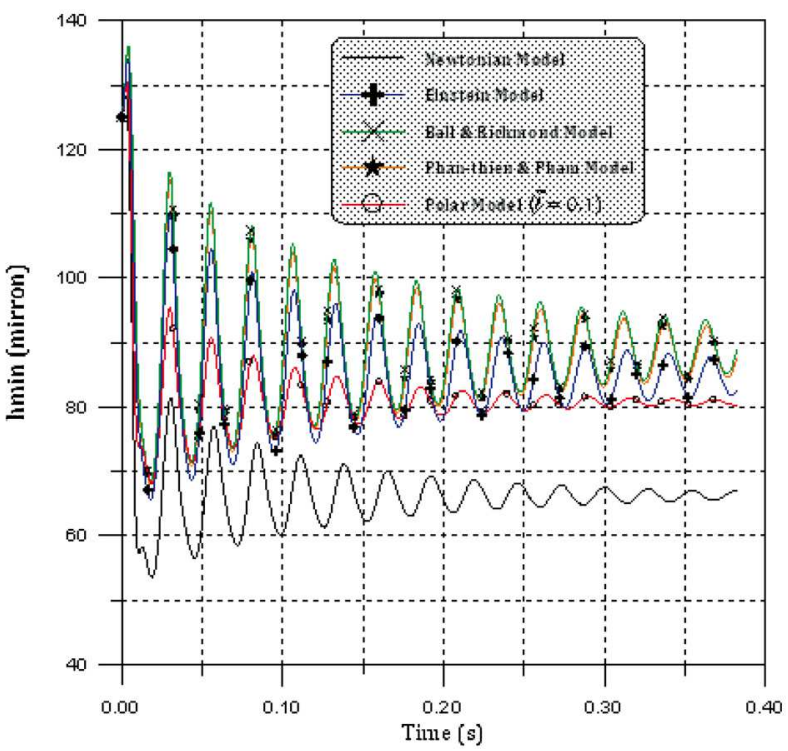

Fig. 9. Minimum film thickness evolution with time for $10 \%$ particle concentration.

It is well known that when the attitude angle increases the bearing stiffness (fluid stiffness) decreases and thus can lead to instability. The instability in this case means that the shaft do not retrieve its equilibrium position and thus can have an orbital or divergent orbit.

\section{Discussion and conclusions}

Starting from a simple approach based on the Einstein theory of particle concentration, we have found that the presence of particles enhances pressure magnitude and load carrying capacity. Likewise, the particles decrease the

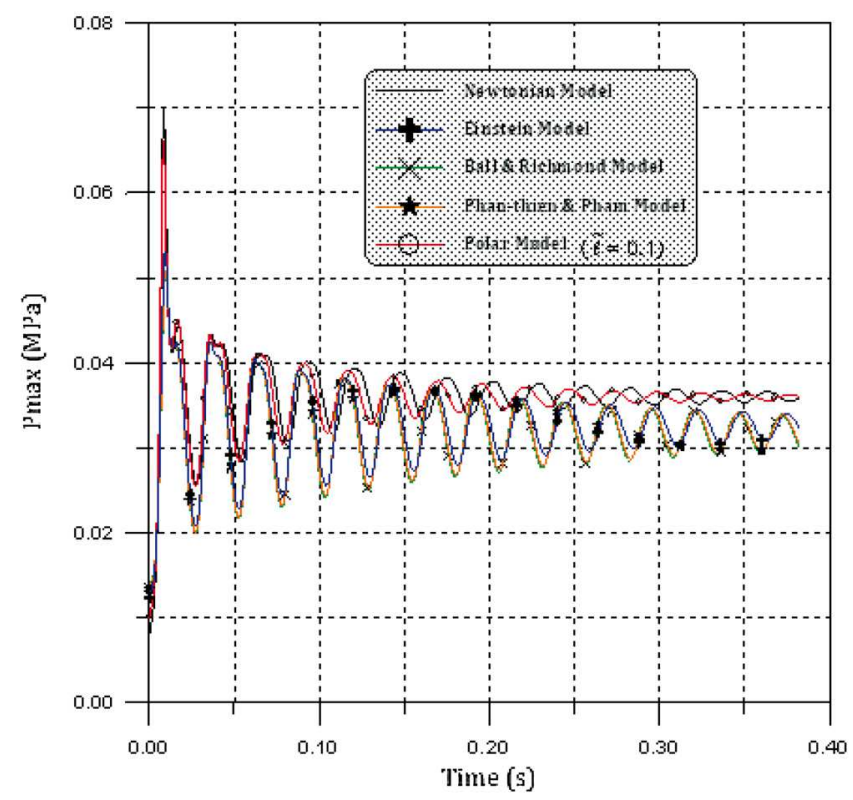

Fig. 10. Maximum pressure evolution with time for $10 \%$ particle concentration.

friction factor, however, no changes are observed in the attitude angle. The nonlinear study shows that when the concentration of particles increases the bearing behavior can become unstable. These are rough estimations or qualitative tendencies.

Further to obtained results it is clear that the addition of particles or molecules in a solvent improves the static characteristics of a hydrodynamic bearing but can lead to dynamic instabilities. In term of predictive service this can be an indicator to inform about the state of health of the mechanism and the detection of a critical concentration which is harmful in term of dynamic reaction but 


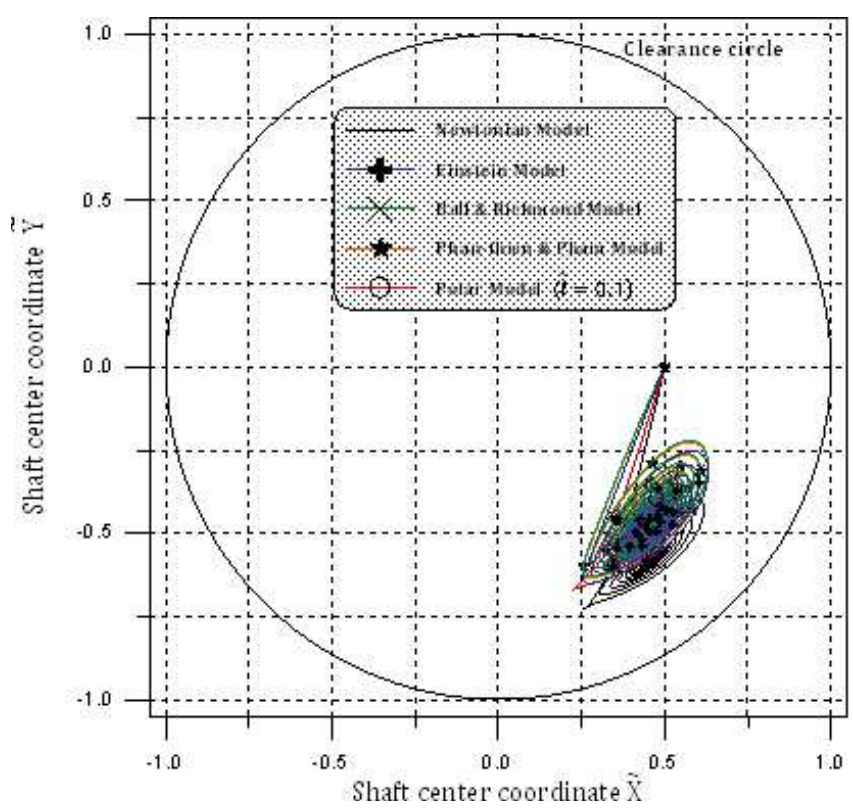

Fig. 11. Shaft trajectories for $10 \%$ concentration of particles.

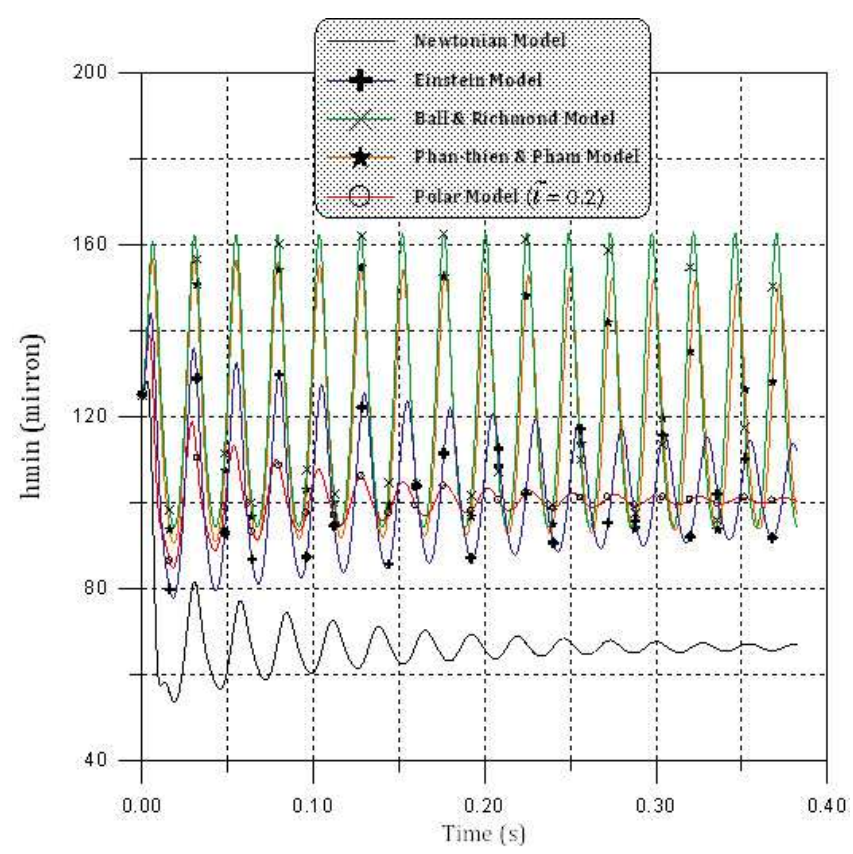

Fig. 12. Minimum film thickness evolution with time for $20 \%$ particle concentration.

also consequently on flow (aggregation leading to one under feeding).

It is clear that in any machine with an oil cycle system, the oil is always in the condition of input, output, flow and stir, and the particles generated by friction and wear are suspended in the oil. Obviously, the particles in the oil are generated and removed continuously when the machine is running. The main mechanisms for the removal of the particles are filtration, settling, impaction and adhesion, combination, dissolution, magnetic separation, etc. The concentration of particles cannot be steady

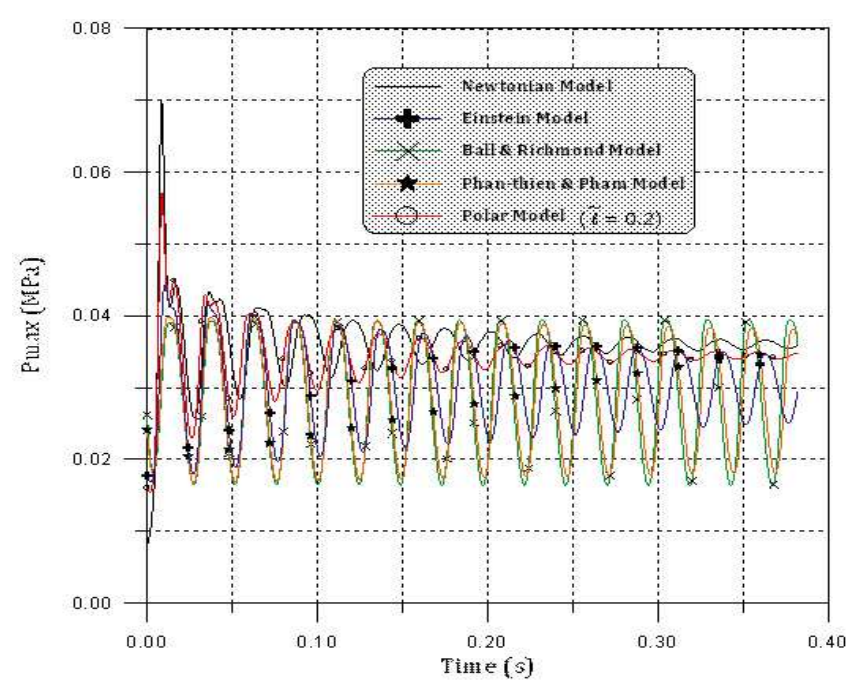

Fig. 13. Maximum pressure evolution with time for $20 \%$ particle concentration.

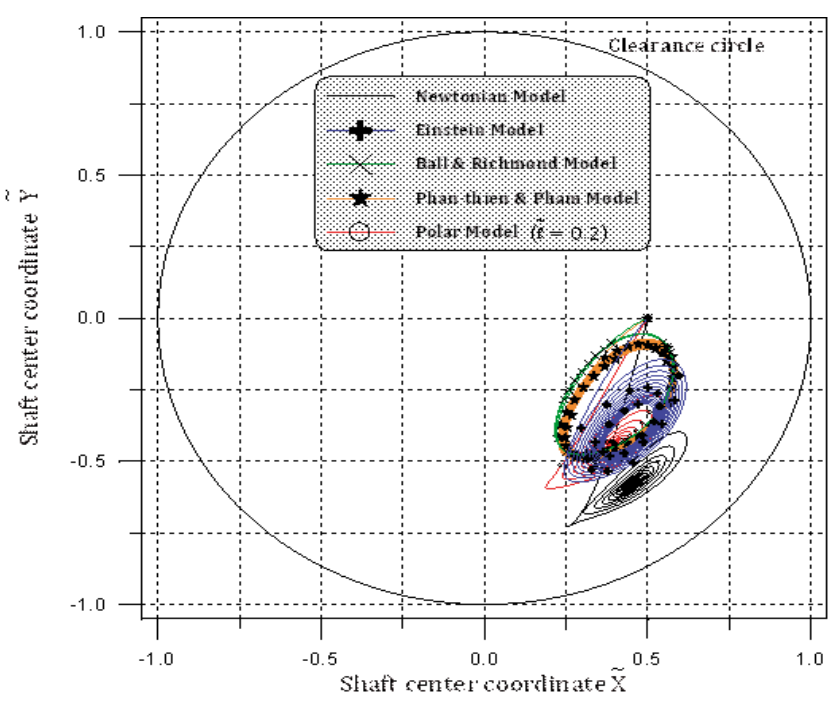

Fig. 14. Shaft trajectories for $20 \%$ particle concentration.

state. A fundamental problem common to all of these conditions is the behavior of the particles in the liquid. It has been shown that the larger the particle diameter and mass concentration, the higher the load carrying capacity. This result was explained in that for a given concentration, finer particles will more easily form a complete and continuous film than coarser particles.

One can develop more sophisticated theoretical approaches. For instance, the Discrete Element Method (DEM) can help in the understanding of such complex phenomenon. The DEM can treat the microscopic particle behavior by solving Newton's equations of motion for each particle. It has become a popular tool for granular material simulation and has been applied to a variety of problems. The soft sphere model, which was first proposed by Cundall and Struck [12], was introduced to gas-solid systems by Tsuji [13] in 1993. 


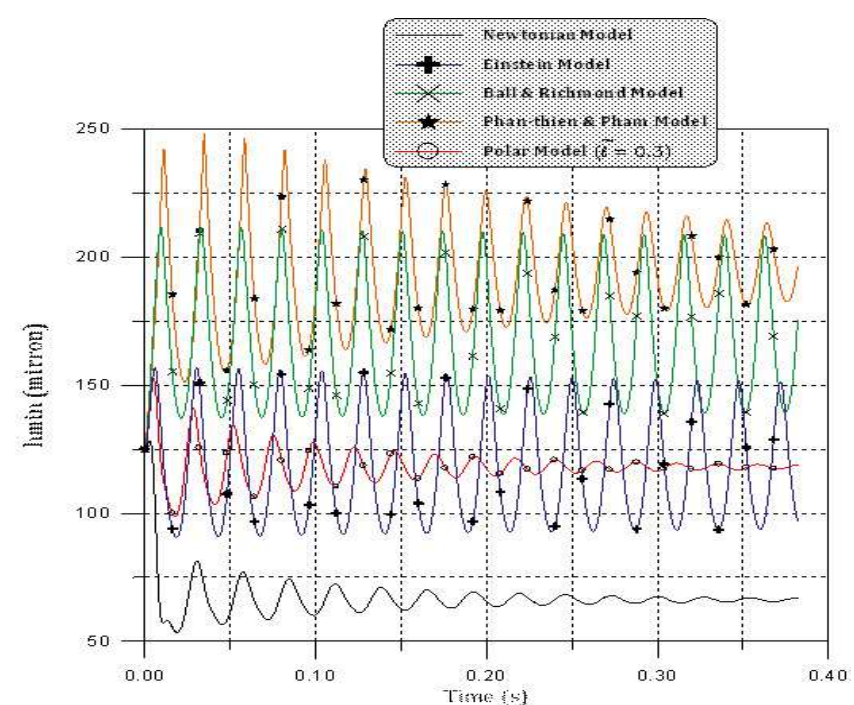

Fig. 15. Minimum film thickness evolution with time for $30 \%$ particle concentration.

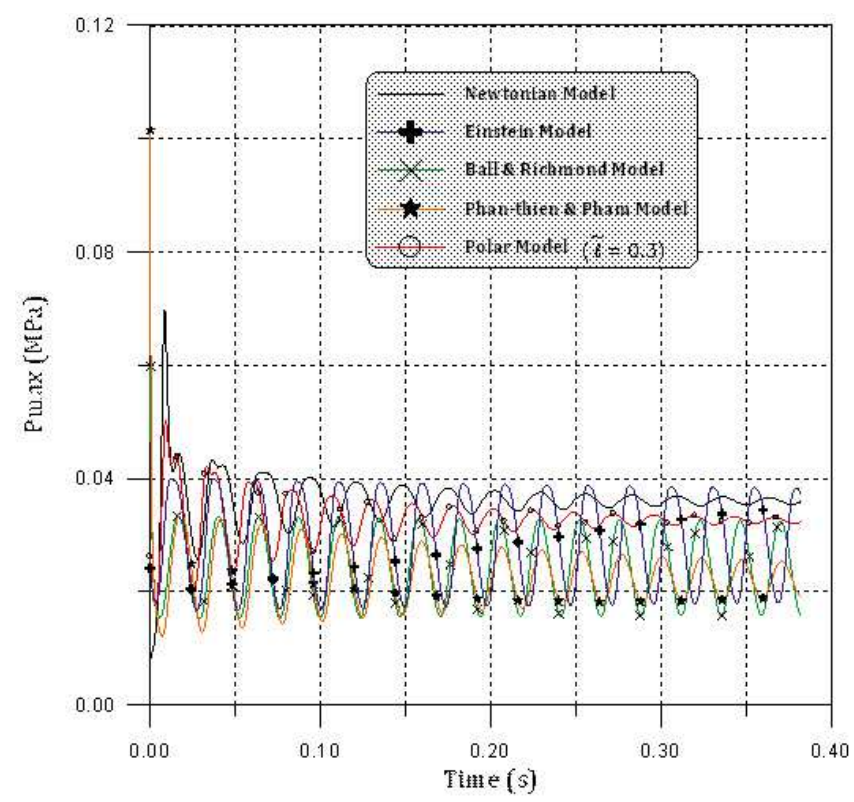

Fig. 16. Maximum pressure evolution with time for $30 \%$ particle concentration.

Subsequently, Horio et al. [14-19] successfully applied the method to solve industrial issues relevant to fluidized beds for particle agglomeration, and combustion with immersed tube and polymerization. However, considerable empiricism still remains as to how to plausibly include different interparticle forces in Newton's equation of motion, and also in how to adjust values such as the coefficient of restitution and the spring constant. To develop DEM further for more realistic simulation, a more accurate and practical collision model should be constructed in which the lubrication effect is included.

As an alternative approach, using the Stokes microcontinuum theory, the effects of couple stress on the static and dynamic response have been presented. To take into

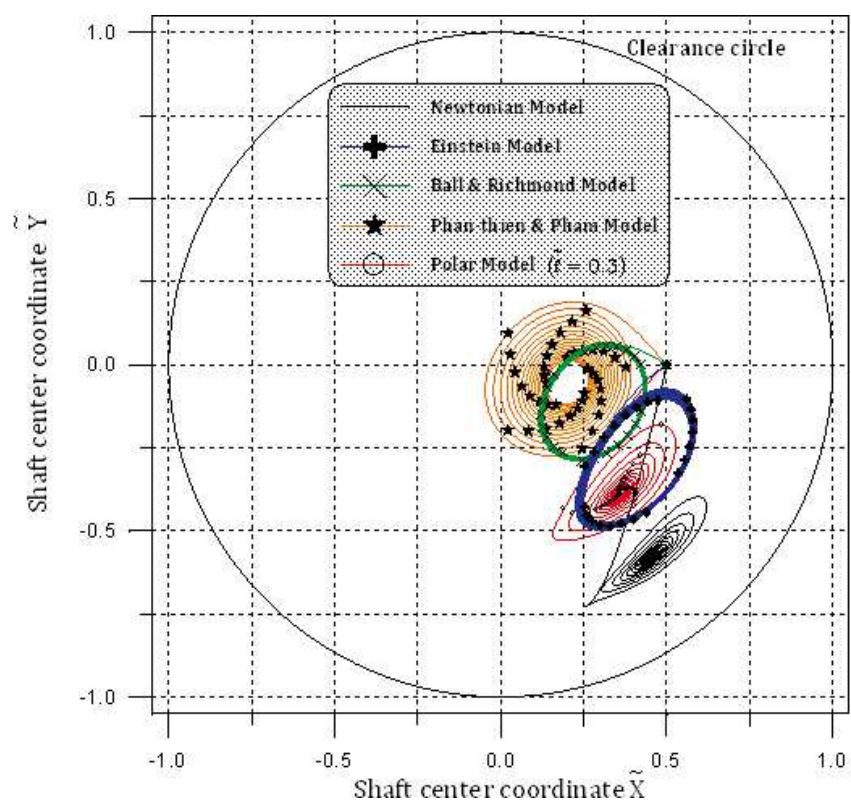

Fig. 17. Shaft trajectories for $30 \%$ particle concentration.

account the couple stress effects due to a Newtonian lubricant blended with particles, a modified Reynolds equation is derived using the Stokes constitutive equations. According to the Stokes theory, a new material constant [12] is responsible for the couple stress property. The parameter $\ell$ may be identified as a characteristic length of a particle in a Newtonian lubricant.

The influence of couple stresses on the bearing characteristics is significant. Compared to the Newtonianlubricant case, the effects of the couple stresses provide an enhancement to the load-carrying capacity, as well as a reduction in the attitude angle and the friction coefficient. The quantitative effects are more pronounced for bearings operating at higher values of eccentricity ratio. The effects of couple stresses due to the lubricant with particles indicate potential beneficial effects on load enhancement and the friction reduction of a lubrication system. This influence agrees qualitatively with the experimental works by Scott and Suntiwattana [20] and Oliver [21]. This latter theoretical approach seems to be closer to experimental investigations. Theoretically speaking, the question as to an appropriate rheological law remains open.

\section{References}

[1] D.R. Oliver, Load enhancement effects due to polymer thickening in a short model journal bearing, J. NonNewtonian Fluid Mech. 30 (1988) 185-196

[2] R.A. Lionberger, Viscosity of bimodal and polydisperse colloidal suspensions, Phys. Rev. E 65 (2002) 061408

[3] R.F. Probstein, M.Z. Sengun, T.-C. Tseng, Bimodal model of concentrated suspension viscosity for distributed particle sizes, J. Rheol. 38 (1994) 811

[4] V.K. Stokes, Couple-stresses in fluids, Phys. Fluids 9 (1966) 1709-1715 
[5] T.T. Ariman, N.D. Sylvester, Micro-continuum fluid mechanics: A Review, Int. Eng. Sci. 11 (1973) 905-930

[6] T.T. Ariman, N.D. Sylvester, Application of Microcontinuum, Int. Eng. Sci. 11 (1974) 905-930

[7] M.U. Mokhiamer, W.A. Crosby, H.A. El-Gamal, A Study of a journal bearing lubricated by fluids with couple-stress considering the elasticity of the liner, Wear 224 (1999) 194-201

[8] R. Tanner, Engineering Rheology, Oxford Engineering Science Series, 2nd edition, 2000

[9] R.B. Bird, R.C. Amstrong, O. Hassager, Dynamics of polymeric liquids, Fluid Mechanics, John Wiley \& Sons Edition, 1987, Vol. 1

[10] H. Boucherit, M. Lahmar, B. Bou-Saïd, Misalignment effects on steady state and dynamic behaviour of compliant journal bearings lubricated with couple stress fluids, Lubrication Science 20 (2008) 241-268

[11] P.D. Cundall, O.D.L. Struck, A discrete numerical model for granular assemblies, Geotechnique 29 (1979) 47

[12] Y. Tsuji, T. Kawaguchi, T. Tanaka, Discrete particle simulation of twodimensional fluidized bed, Powder Technol. 77 (1993) 79

[13] T. Mikami, H. Kamiya, M. Horio, Numerical simulation of cohesive powder behavior in a fluidized bed, Chem. Eng. Sci. 53 (1998) 1927

[14] Y. Kaneko, T. Shiojima, M. Horio, DEM simulation of fluidized beds for gas-phase olefin polymerization, Chem. Eng. Sci. 54 (1999) 5809
[15] D. Rong, T. Mikami, M. Horio, Particle and bubble movements around tubes immersed in fluidized beds : a numerical study, Chem. Eng. Sci. 54 (1999) 5737

[16] K. Kuwagi, T. Mikami, M. Horio, Numerical simulation of metallic solid bridging particles in a fluidized bed at high temperature, Powder Technol. 109 (2000) 27

[17] K. Kuwage, K. Takano, M. Horio, The effect of tangential lubrication by bridge liquid on the behavior of agglomerating fluidized beds, Powder Technol. 113 (2000) 287

[18] D. Rong, M. Horio, Behavior of particles and bubbles around immersed tubes in a fluidized bed at high temperature and pressure: a DEM simulation, Int. J. Multiph. Flow 27 (2001) 89

[19] W. Scott, P. Suntiwattana, Effect of oil additives on the performance of a wet friction clutch material, Wear 181183 (1995) 850-855

[20] G. Bossis, John F. Brady, Dynamic simulation of sheared suspensions, I. General method J. Chem. Phys. 80 (1984) 5141

[21] R.B. Bird, R.C. Amstrong, O. Hassager, Dynamics of polymeric liquids, Kinetic Theory, John Wiley \& Sons Edition, 1987, Vol. 2

[22] B. Bou-Saïd, Quelques problèmes d'écoulements nonlinéaires en lubrification et leur traitement par ElémentsFinis, Revue Européenne des Éléments Finis 10 (2001) $741-753$ 